\title{
Dienst am Menschen, Erfolg in der Forschung
}

\author{
Basels Medizingeschichte bietet interessante Facetten. Am Rheinknie wurden in vielen \\ medizinischen Teilbereichen wichtige Entwicklungsschritte gemacht. Eine Auswahl \\ davon präsentiert die Ausstellung «Dienst am Menschen. Basler Medizingeschichten \\ 1860-2010» zum 150. Geburtstag der Medizinischen Gesellschaft Basel.
}

Thilo Mangold

Korrespondenz:

lic. phil. Thilo Mangold

dill, duss + miozzari

Grünpfahlgasse 8

Postfach

CH-4051 Basel

thilo.mangold@d-d-m.ch

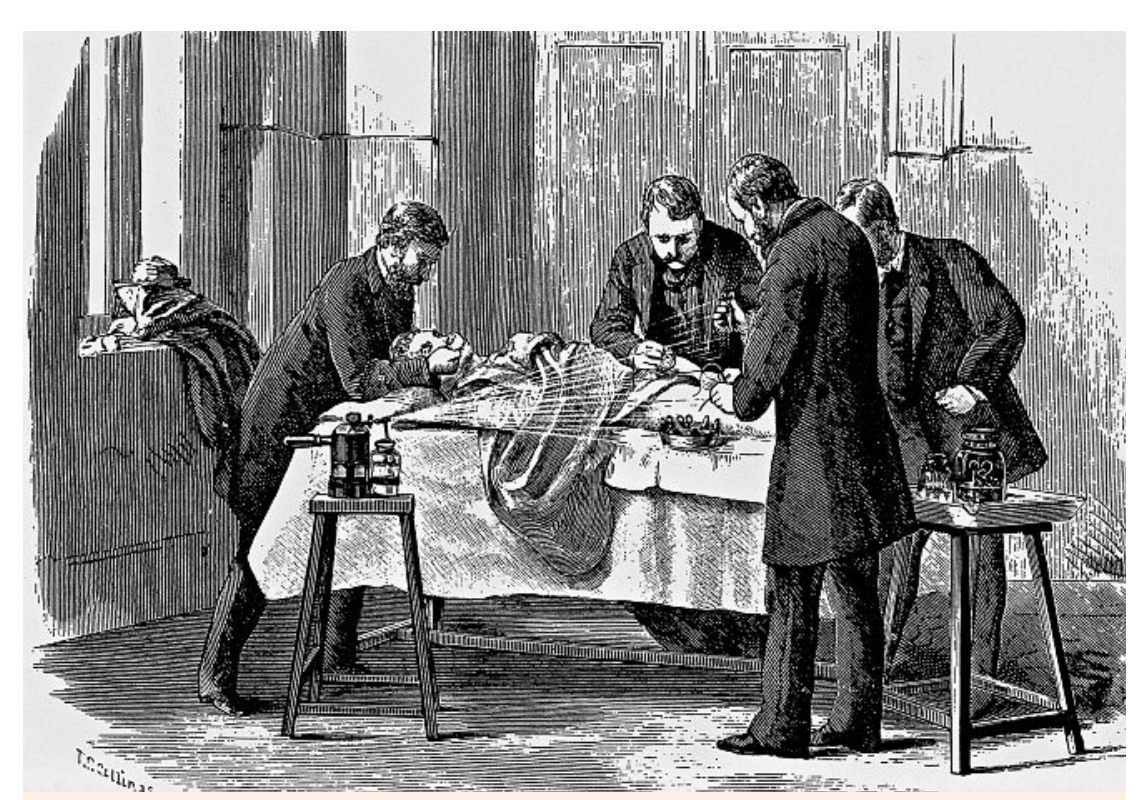

Die Einführung der antiseptischen Wundbehandlung in der zweiten Hälfte des 19. Jahrhunderts bedeutete einen Quantensprung in der Entwicklung der Medizin. Die Jubiläumsausstellung der MedGes zeigt unter anderem, wie August Socin, Chirurg und Mitbegründer der MedGes, an der Etablierung dieser medizinischen Errungenschaft beteiligt war. (Bild: Holzschnitt aus der 1882 veröffentlichten «Antiseptic Surgery» von William Watson Cheyne)

Die Medizinische Gesellschaft Basel (MedGes) wurde 1860 von 18 Ärzten ins Leben gerufen - und begleitet seither als Berufsverband der im Kanton Basel-Stadt tätigen oder wohnhaften Ärztinnen und Ärzte die Entwicklungen der modernen Medizin. Ihre Aufgabe sieht die MedGes darin, die Stellung ihrer Mitglieder in ihrem beruflichen Umfeld zu stärken und sie in der Ausübung ihres Berufes zu fördern und zu unterstützen. Zum 150-Jahr-Jubiläum der Gesellschaft findet in Basel ab dem 20. November eine Ausstellung statt. Der Historiker Christoph Schön hat während der Recherche für die Ausstellung «interessante Verknüpfungen» hergestellt: «Die gesellschaftlichen und juristischen Bedingungen spielten oft eine entscheidende Nebenrolle, wenn der Basler Medizin ein Forschungserfolg gelang.» Die Ausstellung veranschaulicht diese historischen Situationen in ihren Bereichen zur Nierentransplantation oder zur Entstehung der Pharmaindustrie.

(1)

\section{Der Weg zur modernen Medizin}

Seit 150 Jahren setzt sich die MedGes dafür ein, dass Ärztinnen und Ärzte ihren Dienst am Menschen unter den Rahmenbedingungen leisten können, die sie für die besten halten. Das so entstandene und gepflegte Umfeld ermöglicht immer wieder Exploits in der Forschung. Die Ausstellung im Museum Kleines Klingental zeigt eine Selektion davon. Anstelle einer lückenlosen Darstellung der Institutionengeschichte der MedGes blickt die Jubiläumsausstellung auf 150 Jahre allgemeines medizinisches Schaffen am Rheinknie zurück. Themen aus der Gründerzeit der modernen Medizin, wie die antiseptische Wundbehandlung, kommen dabei ebenso zur Sprache wie die Basler Basket-Studie, die vor wenigen Jahren grosse Wellen geschlagen hat. Unkonventionelle Lösungsansätze phantasievoller Basler Ärzte werden museal aufbereitet. Ausstellungsmacher Christoph Schön erwähnt die Tatsache, dass Innovationen, die

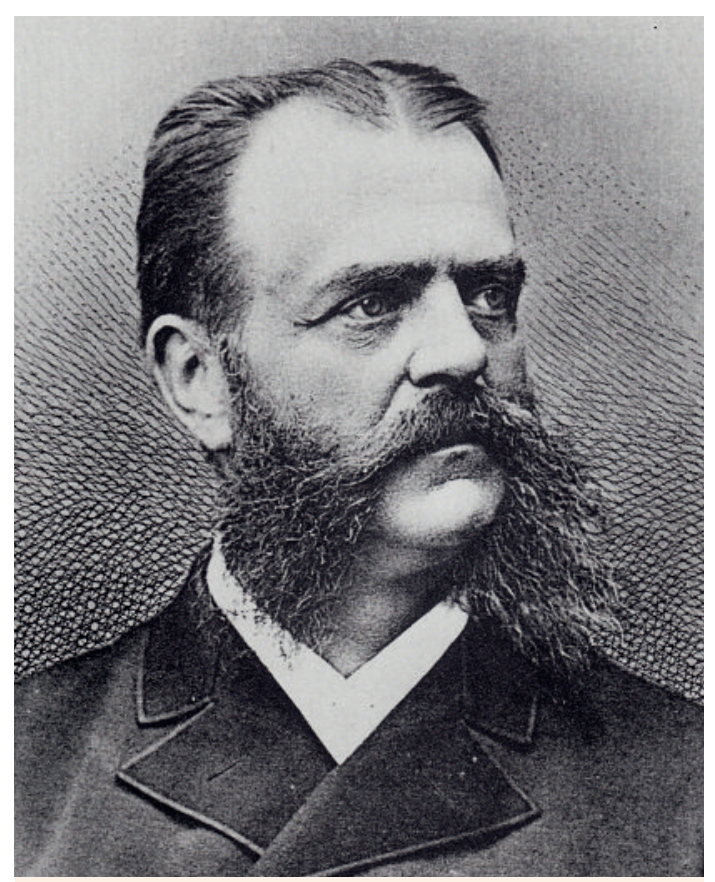

August Socin (1837-1899), Basler Chirurg und Gründungsmitglied der MedGes, war ein Pionier der antiseptischen Wundbehandlung. 


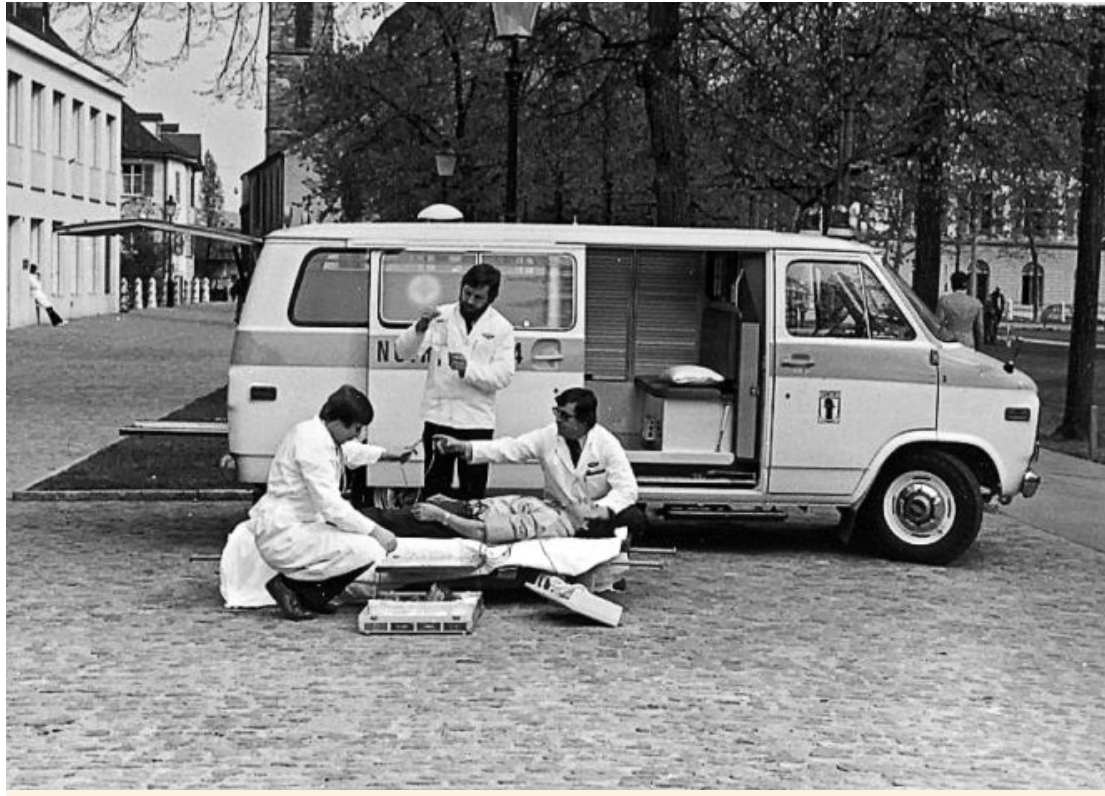

Wichtige Basler Innovation: Das 1972 geschaffene «Kardiomobil» ist eine mobile Herzstation ein Rettungswagen mit Arzt und Defibrillator. (Bild: Bruno Müller, Basler Rettungsdienst)

aus heutiger Sicht selbstverständlich erscheinen, im jeweiligen historischen Kontext «oft kritisch betrachtet wurden und sich gegen Widerstände behaupten mussten». Quer durch alle Teilbereiche der Medizin stellt die Ausstellung «Dienst am Menschen» kleinere und grössere Innovationen aus Basel vor.

\section{Stöbern, staunen, schmunzeln}

Die Ausstellung erhebt keinen Anspruch auf Vollständigkeit. Sie zeigt aber eindrücklich, dass Medizin auf hohem Niveau in Basel Tradition hat - woran die

Führungen zur Ausstellung:

15. 12. 2010, $14.30 \mathrm{Uhr}$

Oskar Kreis - verkannter Pionier der Spinalanästhesie (Prof. Dr. med. Markus Schneider, Universitätsfrauenklinik Basel)

22. 12. 2010, $14.30 \mathrm{Uhr}$

Erfolgsstory Memory Clinic Basel (Doris Ermini, Geronto- und Neuropsychologin und ehemalige Leiterin der Basler Memory Clinic)

9. 1. 2011, $11.00 \mathrm{Uhr}$

Augenpathologie und Kunst (Prof. Dr. med. Peter Meyer, Ophthalmopathologie Augenklinik, Universitätsspital Basel)

26. 1. 2011, 14.30 Uhr und 6. 3. 2011, 11.00 Uhr

Kammerflimmern (Dr. med. Thomas Cron, Facharzt FMH für Kardiologie)

9. 2. 2011, $14.30 \mathrm{Uhr}$

Basel und die Pharmazie (Dr. phil. II Michael Kessler, Leiter Pharmazie-Historisches Museum der Universität Basel)

\section{Dienst am Menschen \\ Basler Medizingeschichte 1860-2010}

Ausstellung «150 Jahre Medizinische Gesellschaft Basel»

20. November 2010 bis 29. Mai 2011

Öffnungszeiten: Mittwoch und Samstag 14.00-17.00 Uhr, Sonntag 10.00-17.00 Uhr

\section{Eintritt frei}

Museum Kleines Klingental,

Unterer Rheinweg 26, 4058 Basel

MedGes nicht unbeteiligt ist. Bei manchen der vorgestellten gesundheitswissenschaftlichen Innovationen hatten Mitglieder der MedGes ihre Finger direkt im Spiel. Bei anderen wiederum war die Gesellschaft hinter den Kulissen beteiligt, immer aber bewegten sich die Protagonisten der Ausstellungsgeschichten in ihrem Umfeld. Die Ausstellung richtet sich mit ihrem erfrischenden Design an ein breites Publikum. Der inhaltliche Zugang erfolgt gewollt niederschwellig und kurzweilig. So wird die Etablierung der Arthroskopie durch die Muttenzer Rennbahnklinik am «Knie der Nation» des Skifahrers Pirmin Zurbriggen veranschaulicht. Oder es wird unter dem Titel «Kokain im Kreisssaal» über die Geschichte der Unterleibsanästhesie berichtet. Staunen und Schmunzeln liegen auf dem musealen Rundgang nahe beieinander. Die Nähe medizinhistorischer Entwicklungen zu Basel und seiner MedGes wird verständlich dargestellt. Die Nordwestschweiz ist in den vergangenen 150 Jahren zu einem wichtigen Standort für medizinische (Neu-) Entwicklungen geworden - mit Mut, Beharrlichkeit und Glück.

\section{2. 2011, $11.00 \mathrm{Uhr}$}

Der grüne Star (PD Dr. med. Konstantin Gugleta, Oberarzt Augenklinik, Universitätsspital Basel)

23. 3. 2011, $14.30 \mathrm{Uhr}$

Pharmazie und Desinfektionsmittel (Dr. phil. II Michael Kessler, Leiter Pharmazie-Historisches Museum der Universität Basel)

10. 4. 2011, $11.00 \mathrm{Uhr}$

Nephrologie: Nierentransplantationen in Basel (Dr. Felix Burkhalter, Transplantationsimmunologie und Nephrologie, Universitätsspital Basel)

22. 5. 2011, $11.00 \mathrm{Uhr}$

Das Knie der Nation: Arthroskopie an Pirmin Zurbriggen (Dr. Bernhard Segesser, Rennbahnklinik Muttenz)

Weitere Führungen und Veranstaltungen zur Ausstellung siehe Tagespresse und www.mkk.ch. Privatführungen nach Vereinbarung (Tel. 07930300 82). 